\title{
Fatal opioid and cocaine related overdoses - Prevention and harm reduction in the light of socioeconomic inequality
}

\section{Alain ORIGER ${ }^{1,2}$ \& Michèle BAUMANN ${ }^{1}$}

\author{
1 University of Luxembourg, research Unit INSIDE, Institute Health \& Behaviour, Walferdange, Luxembourg. \\ 2 Ministry of Health, Luxembourg, Luxembourg.
}

Objective: To determine socio-economic inequalities in fatal overdose cases related to non-prescribed opioids' and cocaine use in the framework of prevention and harm reduction planning.

Case definition. Death cases registered nationally between 1994 and 2011 (n: 272) for which an acute adverse somatic reaction after the recent use of products containing opiates and/or cocaine is reported as the primary cause of death in the light of available toxicological and forensic evidence.

Data collection and sources. Compilation and crossexamination of data from law enforcement sources, the national drug use surveillance system (RELIS) and of forensic and toxicological evidence.

Study design. Overdose cases recorded between 1994 and 2011 were individually matched with four controls, in a nested case-control study design, according to sex, year of birth, drug administration route and duration of drug use. 272 cases vs. 1,056 controls were analyzed. The study sample was stratified according to a Social Inequality Accumulation Score (SIAS), based on educational attainment, employment, income, financial situation and professional status of the father or the legal guardian of subjects.

Statistical analyses. Weighted least squares linear regression analysis on overdose mortality rates and ridit scores were applied to determine the Relative Index of Inequality (RII). In order to account for missing values, we performed multiple imputations using the fully conditional specification approach on the entire data set.

Results: Fatal overdose victims concentrate in lower SIAS classes. The mean SIAS of victims is 1.22 , compared to 1.85 for survivors ( $t=25.089 ; \mathrm{p}=.000$ ).

A negative linear relationship between the overdose mortality rate and the relative socioeconomic position was observed. We found a difference in mortality of 29.22 overdose deaths per 100 drug users between the lowest socioeconomic class, compared to the most advantaged class.

In terms of Relative Inequality Index (RII), the overdose mortality rate of opioid and cocaine users with lowest socioeconomic profiles was 9.88 times higher than in their peers from the highest socioeconomic class [RII: 9.88 (95\% CI 6.49 - 13.26)].
Table 1. Overdose mortality rates and ridit scores associated to SIAS classes

\begin{tabular}{|c|c|c|c|c|}
\hline $\begin{array}{c}\text { SIAS } \\
\text { class* }\end{array}$ & $\begin{array}{c}\text { Overdose } \\
\text { Mortality } \\
\text { Rate (OMR) }\end{array}$ & $\begin{array}{c}\text { N } \\
\text { survivors }\end{array}$ & $\begin{array}{c}\text { Relative } \\
\text { frequency } \\
\text { (RF) }\end{array}$ & Ridit $®$ \\
\hline$\leq 1$ & 0.29288 & 283 & 0.31152648 & 0.15576 \\
\hline $\begin{array}{c}>1 \text { and } \\
\leq 2\end{array}$ & 0.17074 & 325 & 0.305373832 & 0.46421 \\
\hline $\begin{array}{c}>\text { and } \\
\leq 3\end{array}$ & 0.10550 & 272 & 0.236760125 & 0.73528 \\
\hline$>3$ & 0.07806 & 173 & 0.146339564 & 0.92683 \\
\hline
\end{tabular}

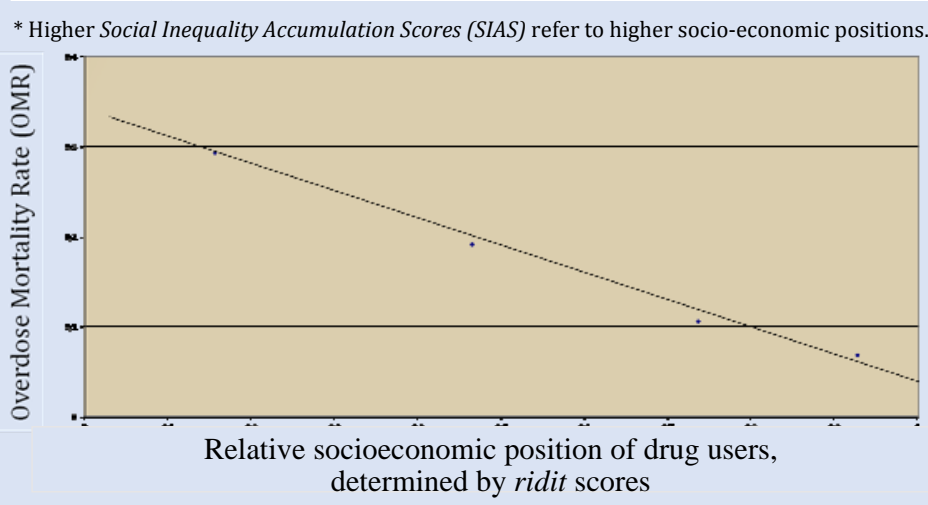

Figure 1. Overdose Mortality Rate (OMR) according to relative socioeconomic positions determined by ridit scores associated to SIAS.

Conclusions: Our results suggest the existence of a marked social gradient in opioid and cocaine related overdose fatalities. In terms of prevention and harm reduction strategies, this is a relevant finding as it suggests that any measure aiming at reducing social disparities, even if isolated or targeted, may have a positive and dynamic impact that counter the negative cumulative effect in terms of survival. This strongly supports the idea that drug-related harm reduction strategies exclusively focusing on reducing direct harm of drug use fail to address harm associated with the context of drug use such as homelessness, violence and poverty . Medical services, emergency and psychiatric wards should take due account of the socio-economic situation of substance misusing patients and refer those at increased risk to socially supportive offers, thus contributing to reduce drug-related mortality.

Acknowledgements: Thanks are due to the Department of Toxicology of the National Laboratory of Health for providing forensic data and the staff of the EMCDDA Focal Point Luxembourg (Luxembourg Institute for health) for its relentless efforts to ensure and maintain the quality of data provided by the national RELIS surveillance system and to all involved agencies. 